\title{
Research Grade Remote-Access Scanning Electron Microscopy: The Evolution of the Bugscope Project
}

\author{
Glenn Fried, Scott Robinson, Don Appleman, Ben Grosser, Daniel Weber, and Chas Conway
}

Imaging Technology Group, Beckman Institute for Advanced Science and Technology, University of Illinois, Urbana, IL, 61801

The Bugscope Project, launched in March 1999, is an educational outreach program designed to give kindergarten through 12th grade students throughout the United States (and other countries when feasible) access to and control of a field emission environmental scanning electron microscope (ESEM) using web browsers in their own classrooms. With the active participation of their teachers, classrooms apply for connection time over the web and may mail in insect or small arthropod specimens to be prepared for their sessions. The specimens are mounted on a 50-mm stub, coated with gold-palladium, grounded with silver paint, and placed in the ESEM chamber for imaging. Eight preset positions, in $\mathrm{x}, \mathrm{y}, \mathrm{z}$ (focus), and with the appropriate brightness/contrast, intended to pique the students' interest and give them starting points from which to explore the sample stage, are defined in the software before each session. The preset positions are available to the students on the controller window. During the Bugscope session, a number of observer and chat windows may be opened on classroom computers; students can take turns operating the microscope using a single controller window. Each command from the classroom's controller results in the requested change being performed on the microscope and the resulting image appearing on the observer window. Each image resulting from each command, good or bad, is stored on a webaccessible database under the name of the school operating the electron microscope at the time. The chat window has proven to be beneficial: laboratory personnel handle student and teacher queries ranging from how to run the controller to insect anatomy/facts to the theory of electron microscopy and "what it's like to be a scientist." Specific details and statistics relating to the project have been discussed previously $[1,2,3]$. The project has recently surpassed 110 actual sessions, in addition to numerous demonstration and test connections from as far away as The Netherlands and Australia.

With remote control sessions now taking an average of 100 hours per year, we have written a new research grade application for controlling the ESEM. Our goal is to provide a tool that can be used by engineers and scientists nationwide who require access to a field-emission environmental scanning electron microscope. Uses for this type of tool include collaborative research projects, laboratory projects for virtual classrooms, specific instrument access for smaller research facilities, and classroom teaching tools.

Software modifications have been made to improve usability. A remote scheduling system has been coupled to our local system to permit participants to reserve time on the instrument. Simplified remote access initialization processes have been applied, enabling students to set up remote sessions in a stepwise manner with few or no complications. Streaming video is now utilized during stage movement to facilitate locating features of interest. Full control of accelerating voltage, spot size, magnification, brightness/contrast, and astigmatism correction has been implemented, along with the ability to record and store extra high definition images. These modifications represent a significant step toward nearly complete remote control of the microscope by dedicated researchers. 
Future changes are intended to make wet mode imaging, backscattered electron imaging, and energy-dispersive spectroscopy as fully operational as possible through a remote connection [4].

\section{References}

[1] U. Thakkar et al., Paper prepared for the Annual Meeting of American Educational Research Association, Special Interest Group on Advanced Technologies for Learning, New Orleans, LA, April 24-28, 2000.

[2] C. S. Potter et al., Paper for the panel on "Participatory science via the Internet", pp.379-380, International Conference of the Learning Sciences 2000 (ICLS 2000).14-17 June 2000, Ann Arbor, Michigan.

[3] C. S. Potter et al., Microsc. Microanal. 7 (2001) 249.

[4] Support for the purchase of the ESEM was provided by NSF (DBI-9871103) and the Beckman Foundation; the Bugscope Project was supported by the Illinois Consolidated Telephone Company, the IBM Shared University Research Program, the Beckman Institute for Advanced Science and Technology, and the University of Illinois.

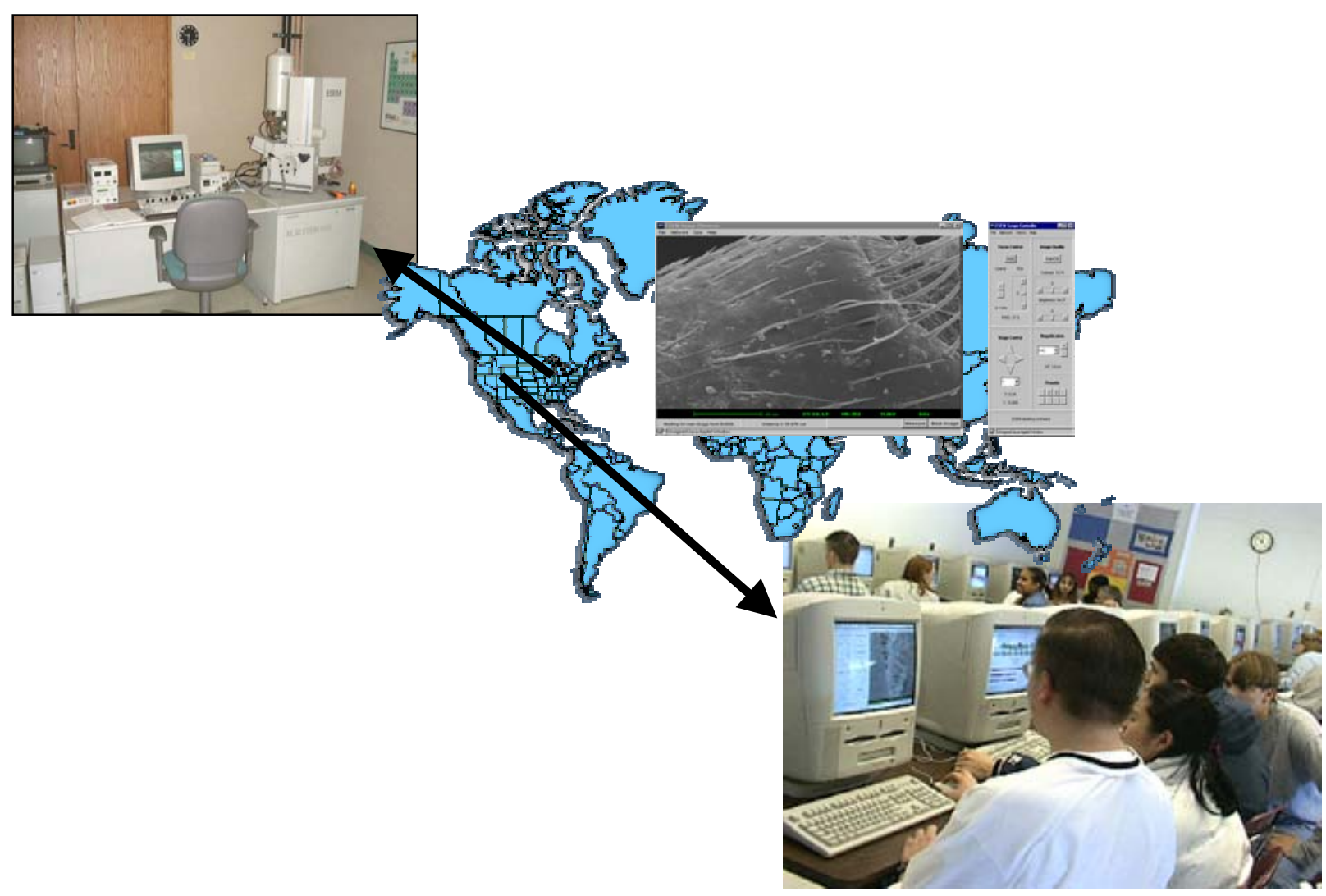

